\title{
Research on a High-Efficiency LED Driving Circuit Based on Buck Topology
}

\author{
Renbo $\mathrm{Xu}^{1,2,3^{*}}$, Hongjian $\mathrm{Li}^{1,2}$, Yongzhi $\mathrm{Li}^{4}$, Xiaomao $\mathrm{Hou}^{3}$ \\ ${ }^{1}$ School of Materials Science and Engineering, Central South University, Changsha, China \\ ${ }^{2}$ School of Physics Science and Technology, Central South University, Changsha, China \\ ${ }^{3}$ Hunan Information Science Vocational College, Changsha, China \\ ${ }^{4}$ College of Physics and Information Science, Hunan Normal University, Changsha, China \\ E-mail: ${ }^{*} x r b 1118 @ 163 . c o m$
}

Received June 22, 2011; revised July 15, 2011; accepted August 10, 2011

\begin{abstract}
A high efficiency LED (Light Emitting Diode) driver based on Buck converter, which could operate under a wide AC input voltage range $(85 \mathrm{~V}-265 \mathrm{~V})$ and drive a series of high power LEDs, is presented in this paper. The operation principles, power loss factors of the LED driver in this study are analyzed and discussed in detail and some effective ways to improve efficiency are proposed through system design considerations. To verify the feasibility, a laboratory prototype is also designed and tested for an LED lamp which consists of 16 LUMILEDS LEDs in series. Experimental results show that a high efficiency of $92 \%$ at $I_{o}=350 \mathrm{~mA}$ can be achieved and the studied driver might be practical for driving high power LEDs. In the last, the overall efficiency over $90 \%$ is gained through some experiments under variable input and output voltages and verifies the validity of the designed driver.
\end{abstract}

Keywords: LED Driver, Buck Type, High Efficiency, Universal Input Voltage

\section{Introduction}

Among the many artificial lighting sources, high power LED characterized by high luminous intensity, superior longevity, cost-effective and less environment impact, is one of the most competitive to replace the conventional incandescent lamp and fluorescent lamp and gradually becomes a commonly used solid-state lighting source in many lighting applications [1-3]. It has been extensively used in the offline lighting field, such as automotive illuminations, liquid crystal display (LCD) backlights and street lighting, where multiple strings of LED driving techniques are often needed. The increased popularity of high power LED has given a requirement for electronic engineers to propose a series of driving circuits with high efficiency and low-cost.

LED dimming control methods could be simply divided into two categories: analog dimming and pulse width modulation (PWM) dimming regulators [4-7]. Analog dimming method is not a good candidate due to colour variation, even though it is simple and cost effective. To avoid colour variation and get accurate current control over full range, PWM dimming regulators in which the pulse current is kept constant.

To research the overall efficiency of LED lighting, a PWM dimming method, Buck converter is chosen in this paper as shown in Figure 1, and the power dissipation factors are analyzed with all the particulars, then a detailed LED driver example with high power efficiency is designed and implemented. In addition to the integrated circuit(IC), the main components mainly includes a power MOS switch $\mathrm{S}$, a Schottky diode $\mathrm{D}$, an inductor $\mathrm{L}$ directly connected with the load LEDs and the current sampling resistor Rs. In fact, the major power dissipation of Buck converter is on these components. Next to make a detail analysis of them.

\section{Operation Principle and Power Loss Analysis}

During the time interval of state 1 , the metal oxide semiconductor field effect transistor (MOS-FET) $\mathrm{S}$ is turned on, current flows through MOS switch S, input inductor L, storage capacitor $\mathrm{C}$, the load LED strings and sampling resistor Rs. The power supply stores energy in the inductor $\mathrm{L}$, the storage capacitor $\mathrm{C}$ and the free-wheeling diode 


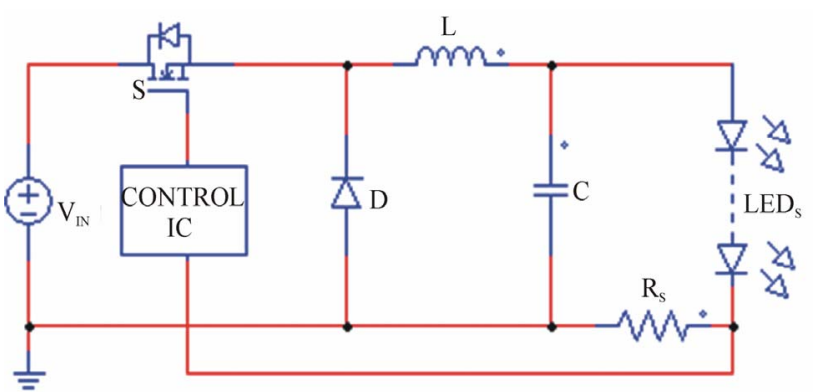

Figure 1. Circuit diagram of Buck converter.

$D$ is off at the moment. During the time interval of state 2, the MOS switch S turns off. The LED strings power is provided by the storage capacitor $\mathrm{C}$, and the energy stored in inductor $\mathrm{L}$ flows through the free-wheeling diode $\mathrm{D}$ simultaneously. Then the circuit proceeds back to stage 1 when the MOS switch $S$ turns on again.

From the analysis it can be known that the power dissipation on the power switch $S$ is described in following Equation (1):

$$
P_{S(\text { on })}=I_{O}^{2} \times R_{S(\text { on })} \times D
$$

where $R_{S(o n)}$ is conduction resistor of MOS switch, $I_{O}$ is the LED strings current and $D$ is the duty cycle of the power switch.

In fact, there is still some power dissipation in the MOS switch when it operates in high frequency. Especially when LED constant-current driving circuit is working in frequency more than $100 \mathrm{kHz}$, this power dissipation is quite substantial which can be expressed as follows [8]:

$$
\begin{aligned}
& P_{S(\text { high-freq })} \\
= & Q_{g s} \times V_{G S} \times f_{s}+\frac{1}{2} \times V_{I N} \times I_{O} \times\left(t_{r}+t_{d}\right) \times f_{s}
\end{aligned}
$$

where $Q_{g s}$ is the MOS gate-source charge and $V_{G S}$ is the MOSFET gate drive voltage, $V_{I N}$ is the input voltage, $t_{r}, t_{d}$ are the needed time of MOS turn-on and turn-off respectively, $f_{s}$ is the switching frequency. From the Equation (2), it is clear that the power dissipation is proportional to the switching frequency. Combining Equation (1) together with Equation (2), we can get the total power dissipation as described in Equation (3):

$$
\begin{aligned}
P_{S}= & P_{S(\text { on })}+P_{S(\text { high-freq })} \\
= & I_{O}^{2} \times R_{S(\text { on })} \times D+Q_{g s} \times V_{G S} \times f_{s} \\
& +\frac{1}{2} \times V_{I N} \times I_{O} \times\left(t_{r}+t_{d}\right) \times f_{s}
\end{aligned}
$$

in order to decrease the total power dissipation, on one hand we should choose a MOS switch with a small conduction resistance, on the other hand the high-frequency characteristic of MOS switch should be taken into consideration if the driving circuit operates in a high frequency.

Inductor $\mathrm{L}$ in the Buck converter which is connected directly to the load LED strings plays an important role of energy storage and transformation. As an LED constant current driver, the Buck converter usually operates in continuous current conduction mode (CCM) so that most of the system power dissipation is consumed on the inductor L. Similar to other switched-mode power supplies, the inductor power dissipation can be divided into two parts: iron loss and copper loss $[9,10]$. Copper loss is determined by the output current and inductor DC resistance, and iron loss caused by eddy current is determined by the switching frequency. The total power consumption of the inductor can be expressed as follows:

$$
P_{L}=I_{O}^{2} \times R_{L(D C)}+P_{c}
$$

where $R_{L(D C)}$ is inductor DC resistance, $P_{c}$ is iron loss of the inductor.

In the LED constant current driver circuit, using a large inductor can effectively reduce the ripple current flowing through the LED strings [11]. However, a problem arises that it would bring greater power loss due to a greater inductor DC resistance. Another method to decrease the ripple current is to parallel a large capacitor with the output LED strings $[12,13]$. But large capacitance means slow frequency response which is not suitable in high precision control requirement occasion.

In the Buck converter, the function of the diode $D$ is to provide a free-wheeling path for the inductor current when MOS switch turns off. Because of high working frequency, a reverse fast recovery Schottky diode has been chosen. When MOS switch is turned-on, the Schottky diode is reverse biased and there is no power dissipation. When the MOS switch is turned-off, the power consumption of free-wheeling diode can be derived by:

$$
P_{D}=I_{O} \times V_{D(\text { on })} \times(1-D)
$$

where $V_{D(o n)}$ is the corresponding forward voltage drop when following through the LED current $I_{O}$.

It could be seen from the Equation (5) that the diode power dissipation is proportional to the forward voltage drop and decreases with the duty cycle increasing. As a matter of fact, the control chip has a limited output duty cycle which makes the diode a minimum power loss. Another way to reduce the power consumption is synchronous rectifier technique $[14,15]$. The diode is replaced by a MOS switch with a low resistance, and the power loss is significantly decreased even if the LED output current is high. However, the synchronous rectification is bound to bring a complicated driving circuit, which needs a balance between performance and cost. 
The last system efficiency factor is the driving chip and the sampling resistor Rs. With the development of LED driving IC, the chip commonly adopts a low comparison voltage about $200 \mathrm{mV}$, so the power consumption of the sampling resistor is not great.

\section{System Design Considerations}

In order to design an efficient LED constant-current driver, a certain process is given as follows about how to select components.

According to the LED constant driving current requirements, a suitable driving chip and a sampling resistor should be selected. Referring to the relevant LED materials of manufacturers, we can get the output voltage under rated current. As mentioned in Equation (6), the corresponding minimum input voltage can be chosen when the chip operating in the allowed maximum duty cycle.

$$
D=\frac{V_{O}}{V_{I N}}
$$

If the chip has no built-in MOS switch, a external MOS switch is needed to choose. Generally speaking, the higher voltage endurance is, the worse the other corresponding characteristics is. Therefore, according to the working environment we should choose a suitable MOS switch with an appropriate voltage endurance and with the conduction resistance and the gate-source charge as an important choice standard. Next to find out the required inductance value calculated by the Equation (7) [16]:

$$
L=V_{O} \times T \times(1-D) / \Delta i
$$

where $T$ is a cycle time of Buck converter, and $\Delta i$ is the allowed maximum ripple current. The inductor should have small DC induction resistance and meet with the frequency requirement. Then, decide whether to use an output capacitor according to the requirements of PWM dimming system and circuit volume. In the case of meeting the ripple current requirements, we should choose a small inductance due to small power consumption.

A suitable diode should be selected according to the reverse bias voltage endurance and its frequency response should be fast enough. Generally speaking, a Schottky diode with low forward voltage drop can be used in most situations blew $100 \mathrm{~V}$. Otherwise, we should choose a fast diode with a high forward voltage drop and a high reverse bias voltage endurance.

\section{Example Design and Experimental Results}

In this section, an LED constant current driver with high power efficiency is designed and implemented according to the following configuration:

- Input voltage: AC 85 - $265 \mathrm{~V}$ (nominal $220 \mathrm{~V}$ )

- LED string voltage: DC $30-60 \mathrm{~V}$

- LED current: $350 \mathrm{~mA}$

- Expected efficiency: 90\%

An LED constant current Buck topology driving IC SL221 from SYNCOAM with a input DC supply voltage range from $9 \mathrm{~V}$ to $550 \mathrm{~V}$ is chosen as a controller and the schematic diagram shown in Figure 2. And it is used to drive an LED string with 16 LUMILEDS LED with a 3.3 $\mathrm{V}$ working voltage.

The AC $220 \mathrm{~V}$ input voltage is converted to $V_{I N}=310 \mathrm{~V}$ after following through the rectifier bridge and the filtering capacitors. The system output voltage is $V_{O}=n \times V_{L E D}=52.8 \mathrm{~V}$ and the duty cycle is 0.17 calculated by Equation (6). High switching frequency will reduce the size of the inductor $L$, but it will increase the circuit's switch dissipation. The oscillator frequency of SL221 is from $20 \mathrm{kHz}$ to $150 \mathrm{kHz}$ and we choice a typical constant switching frequency $40 \mathrm{kHz}$. The timing resistor should be $620 \mathrm{k} \Omega$ and connect to the ground referring to SL221 manual. During the MOSFET switch is on, the gate is charged by high-frequency current pulse. And in order to maintain stability of the internal power supply voltage, C3 a typical capacitance value $2.2 \mathrm{uF}, 16$ $\mathrm{V}$ is recommended.

Next to choose the capacitance values of $\mathrm{C} 1$ and $\mathrm{C} 2$. In order to satisfy the stability requirements at constant switching frequency, the maximum LED string voltage must be less than the half of the minimum input voltage. The minimum input voltage is shown in Equation (8):

$$
V_{I N(\min )}=2 \times V_{O(\max )}=120 \mathrm{~V}
$$

The capacitance value $\mathrm{C} 1$ should be calculated at the minimum AC input voltage, and the nominal voltage should be larger than the input peak voltage as shown in the following Equations (9) and (10):

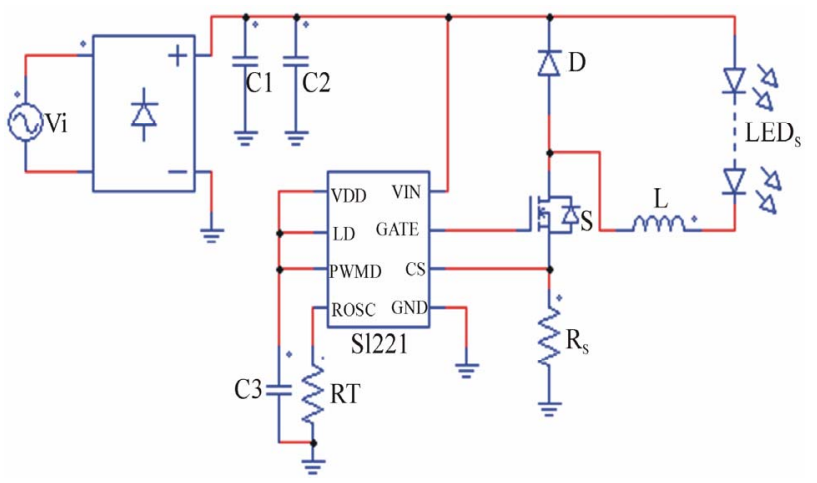

Figure 2. Schematic diagram of the studied LED driver. 


$$
\begin{gathered}
C_{1} \geq \frac{I_{O(\max )} \times V_{O(\max )}}{\left(2 \times V_{i(\min )}^{2}-V_{I N(\min )}^{2}\right) \times f_{s} \times \eta} \Rightarrow C_{1} \geq 11.67 \mu \mathrm{F} \\
V_{C_{1}(\max )} \geq \sqrt{2} \times V_{i(\max )} \Rightarrow V_{C_{1}(\max )} \geq 375 \mathrm{~V}
\end{gathered}
$$

From the mentioned above, we choose a electrolytic capacitor with $33 \mathrm{uF}, 450 \mathrm{~V}$. The stability of the electrolytic capacitor is good. However, it is not suitable for high frequency ripple current absorption which is generated by the Buck converter due to large ESR (Equivalent Series Resistance). So a metalized polypropylene capacitor in parallel with the electrolytic capacitor is used to absorb the high frequency ripple current. The high frequency capacitance $0.47 \mathrm{uF}$ and withstanding voltage $400 \mathrm{~V}$ is determined by the following equation:

$$
C_{2} \geq \frac{0.25 \times I_{O(\max )}}{\left(0.05 \times V_{I N(\min )}\right) \times f_{s}} \Rightarrow C_{2} \geq 0.365 \mu \mathrm{F}
$$

Next to calculate the inductance value which is determined by the permissible ripple current and we assume that the existence of LED ripple current is allowed $\pm 15 \%$ (total ripple is $30 \%$ ). During the MOSFET is off, the inductor supplies energy to the LED string as shown in Equation (12):

$$
E_{L}=V_{\mathrm{LED}}=V_{O(\max )}=L \frac{\mathrm{d} i}{\mathrm{~d} t} \Rightarrow L=V_{O(\max )} \frac{\mathrm{d} t}{\mathrm{~d} i}
$$

where $d i=0.3 \times I_{o, \max }$ is the ripple current, $\mathrm{d} t$ is the off time described as follows:

$$
d t=\frac{1-\frac{V_{O(\max )}}{\sqrt{2} \times V_{i(\text { rated })}}}{f_{s}}
$$

From Equations (12) and (13), the inductance value $L$ can be represented as the following equation:

$$
L=\frac{V_{O(\max )} \times\left(1-\frac{V_{O(\max )}}{\sqrt{2} \times V_{i(\text { rated })}}\right)}{0.3 \times I_{O(\max )} \times f_{s}}
$$

The standard inductance value $4.7 \mathrm{mH}$ is selected which is larger than the calculated value, so the ripple current would be less than $30 \%$. The DC resistance is 3.2 $\Omega$ and the inductor dissipation can be derived by the Equation (4).

MOSFET peak voltage is equal to the maximum input voltage and the safety margin is $50 \%$ described as follows:

$$
V_{\text {MOSFET }}=1.5 \times\left(\sqrt{2} \times V_{i(\max )}\right)=562 \mathrm{~V}
$$

The maximum RMS of MOSFET current depends on the maximum duty cycle, so the nominal current can be expressed as:

$$
I_{\text {MOSFET }} \approx I_{o(\max )} \times \sqrt{0.17}=0.144 \mathrm{~A}
$$

In order to get the minimum switching power dissipation, STD2NM60 with rated voltage $600 \mathrm{~V}$ and rated current $2 \mathrm{~A}$ is selected. Refer to its manual, the conduction resistance is $2.8 \Omega, t_{r}=8 \mathrm{~ns}, t_{d}=25 \mathrm{~ns}$ and $Q_{g s}=1.8 \mathrm{nC}, V_{G S}=7.5 \mathrm{~V}$.

Next to select the diode. Its nominal peak voltage should be equal to MOSFET switch peak voltage and diode average current described as follows:

$$
\begin{gathered}
V_{D}=V_{\text {MOSFET }}=562 \mathrm{~V} \\
I_{D}=0.83 \times I_{O(\max )}=0.291 \mathrm{~A}
\end{gathered}
$$

So we can choose the fast diode STTH1R06 with a forward voltage drop $1 \mathrm{~V}$, rated voltage $600 \mathrm{~V}$ and rated current $1 \mathrm{~A}$, and the switching power dissipation would be low due to the short reverse recovery time $25 \mathrm{~ns}$.

The relationship between the output current and the sensing resistor can be expressed as:

$$
R_{\mathrm{s}}=\frac{0.25}{1.15 \times I_{O(\max )}} \Rightarrow R_{\mathrm{s}}=0.621 \Omega
$$

If the internal threshold voltage is $0.25 \mathrm{~V}$, the result of the above equation is correct. Otherwise, LD pin voltage should be used to replace the internal threshold voltage. In this case, an appropriate standard resistance $0.62 \Omega$ is selected.

From all the above equations, the theory calculated results are: $P_{S}=0.130 \mathrm{~W}, P_{L}=0.412 \mathrm{~W}, P_{D}=0.291 \mathrm{~W}$, $P_{I C}=0.477 \mathrm{~W}, P_{R s}=0.076 \mathrm{~W}$. Combining all the power loss, the theory total power loss is described as:

$$
\begin{aligned}
P_{\text {loss (theory) }} & =P_{S}+P_{L}+P_{D}+P_{I C}+P_{R S} \\
& =1.386 \mathrm{~W}
\end{aligned}
$$

In order to verify the feasibility, a laboratory prototype is designed and tested as shown in Figure 3. The experimental results are expressed as follows:

$$
\begin{aligned}
& P_{\text {in }}=17.38 \mathrm{~W}, P_{\mathrm{LEDs}}=15.97 \mathrm{~W}, \\
& P_{\text {loss(real) }}=1.41 \mathrm{~W}, \eta=92 \%
\end{aligned}
$$

From the theory and experimental results, it can be seen that the theory power loss is almost equal with the measured power loss and the main power dissipation is on diode $D$, inductor $L$ and IC due to high input voltage and the voltage error value between output and input. However, the power dissipation on the MOS switch is not large because of small duty cycle.

In order to confirm the LED driver's universal validity, a series of experiments under variable input and output voltages are tested, and the efficiencies are shown in Figure 4. It is clear that the system efficiency is over the expected value $90 \%$. 


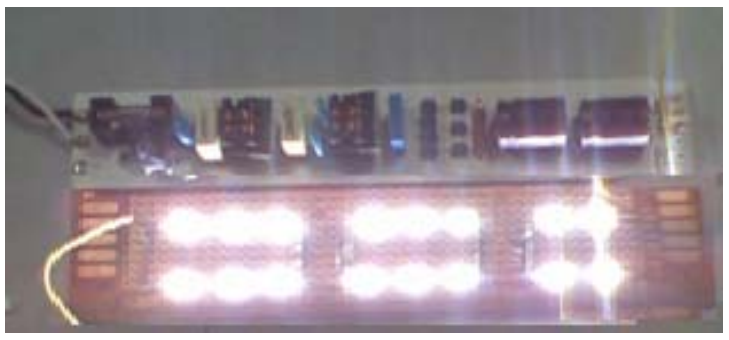

Figure 3. Photograph of the tested prototype driving system.

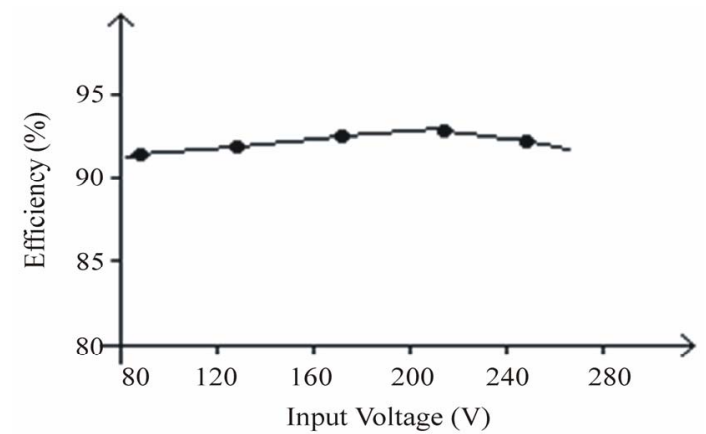

(a)

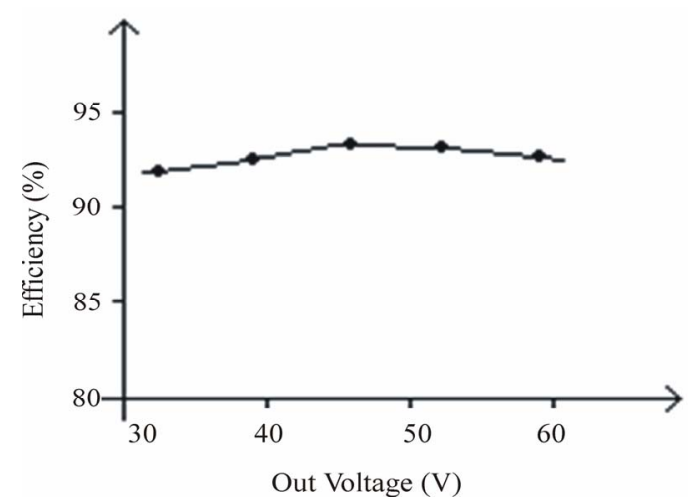

(b)

Figure 4. Measured efficiency vs. input and output voltages. (a) Measured efficiency vs. input voltage; (b) Measured efficiency vs. output voltage.

\section{Conclusions}

LED constant current drive circuit efficiency is determined by several factors, and these factors are interrelated and influenced. To optimize and improve the efficiency of the driving system, the circuit performance and the appropriate components selections must be taken into consideration. In this paper, a high efficiency Buck LED driver with $\mathrm{AC}$ input voltage range from $85 \mathrm{~V}$ to $265 \mathrm{~V}$, which could drive an LED lamp consists of 16 LUMILEDS LEDs, is designed and tested. The measured results on a laboratory prototype show a high efficiency of $92 \%$ at $I_{o}=350 \mathrm{~mA}$. Next, a series of experiments results under variable input and output voltages show that the efficiency is over $90 \%$ and reach the design requirements.

\section{References}

[1] Y. K. Cheng and K. W. E. Cheng, "General Study for Using LED to replace Traditional Lighting Devices," 2nd International Conference on Power Electronics Systems and Applications, Hong Kong, 12-14 November 2006, pp. 173-177. doi:10.1109/PESA.2006.343093

[2] E.-W. Wu, T.-Fu. Wu, J.-R. Tsai, Y.-M. Chen and C.-C. Chen, "Multistring LED Backlight Driving System for LCD Panels with Color Sequential Display and Area Control," IEEE Transactions on Industrial Electronics, Vol. 54, No. 10, 2008, pp. 3791-3800.

[3] M. Rico-Secades, A. J. Calleja, J. Ribas, E. L. Corominas, J. M. Alonso, J. Cardesin and J. Garcia-Garcia, "Evaluation of a Low-Cost Permanent Emergency Lighting System Based on High-Efficiency LEDs," IEEE Transactions on Industry Applications, Vol. 41, No. 5, 2005, pp. 1386-1390. doi:10.1109/TIA.2005.853389

[4] K. H. Loo, W.-K. Lun, S.-C. Tan, Y. M. Lai and C. K. Tse, "On Driving Techniques for LEDs: Toward a Generalized Methodology," IEEE Transactions on Power Electronics, Vol. 24, No. 12, 2009, pp. 2967-2976. doi:10.1109/TPEL.2009.2021183

[5] M. Nishika, Y. Ishizuka, H. Matsuo, et al., "An LED Drive Circuit with Constant Output Current Control and Constant Luminance Control," 28th Telecommunications Energy Conference, Providence, September 2006, pp. 1-6.

[6] H. V. D. Broeck, G. Sauerlander and M. Wendt, "Power Driver Topologies and Control Schemes for LEDs," Twenty Second Annual IEEE of Applied Power Electronics Conference, Anaheim, 25 February-1 March 2007, pp. 1319-1325.

[7] F.-F. Ma, W.-Z. Chen and J.-C. Wu, "A Monolithic Current-Mode Buck Converter with Advanced Control and Protection Circuits," IEEE Transactions on Power Electronics, Vol. 22, No. 5, 2007, pp. 1836-1846. doi:10.1109/TPEL.2007.904237

[8] H.-J. Chiu, Y.-K. Lo, J.-T. Chen, S.-J. Cheng, C.-Y. Lin and S.-C. Mou, "A high-Efficiency Dimmable LED Driver for Low-Power Lighting Applications," IEEE Transactions on Industrial Electronics, Vol. 57, No. 2, 2010, pp. 735-743. doi:10.1109/TIE.2009.2027251

[9] D. Gacio, J. M. Alonso, A. J. Calleja, J. Garcia and M. Rico-Secades, "A Universal-Input Single-Stage HighPower-Factor Power Supply for HB-LEDs Based on Integrated Buck-Flyback Converter," Twenty-Fourth Annual IEEE of Applied Power Electronics Conference and Exposition, Washington, DC, 15-19 February 2009 pp. 570-576.

[10] J. M. Alonso and M. A. Dalla Costa, "Integrated BuckFlyback Converter as a High-Power-Factor Off-Line Power Supply," IEEE Transactions on Industrial Elec- 
tronics, Vol. 55, 2009, pp. 1090-1100. doi:10.1109/TIE.2007.908530

[11] J. A. Villarejo, J. Sebastian, F. Soto and E. de Jodar, "Optimizing the Design of Single-Stage Power-Factor Correctors," IEEE Transactions on Industrial Electronics, Vol. 54, No. 3, 2007, pp. 1472-1482. doi:10.1109/TIE.2007.894734

[12] B. Wang, X. Ruan, K. Yao and M. Xu, "A Method of Reducing the Peak-to-Average Ratio of LED Current for Electrolytic Capacitor-Less AC-DC Drivers," IEEE Transactions on Power Electronics, Vol. 25, No. 3, 2010, pp. 592-601. doi:10.1109/TPEL.2009.2031319

[13] L. Zhao and A. Q. Zhu, "Design of Energy-Saving Intelligent LED Illumination System," Semiconductor Technology, Vol. 33, No. 2, 2008, pp. 137-140.
[14] W. Lin, H. Song, Z. Y. Lu and G. Hua, "A High Efficiency Gate-Driving Scheme of Synchronous Rectifiers in Wide-Input-Voltage-Range CCM Flyback Converter," 37th IEEE Power Electronics Specialists Conference, Jeju, 18-22 June 2006, pp. 1-6.

[15] M. Schmid, D. Kuebrich, M. Weiland, and T. Duerbaum, "Evaluation on the Efficiency of Power LEDs Driven with Currents Typical to Switch Mode Power Supplies," Conference Record of the 2007 IEEE Industry Applications Conference, New Orleans, 23-27 September 2007, pp. 1135-1140.

[16] Y. Hu and M. M. Jovanovic, "LED Driver with SelfAdaptive Drive Voltage," IEEE Transactions on Power Electronics, Vol. 23, No. 6, 2008, pp. 3116-3125. doi:10.1109/TPEL.2008.2004558 